\title{
LMP Congestion Management Using Enhanced STF-LODF in Deregulated Power System
}

\author{
K. B. Ravindra Kumar1*', Dr. S. Chandra Mohan² \\ ${ }^{1}$ Meenakshi College of Engineering, Chennai, Tamil Nadu, India \\ ${ }^{2}$ College of Engineering, Anna University, Chennai, Tamil Nadu, India \\ Email: ravindrakumarphd1@gmail.com
}

Received 1 March 2016; accepted 5 April 2016; published 22 July 2016

Copyright (C) 2016 by authors and Scientific Research Publishing Inc.

This work is licensed under the Creative Commons Attribution International License (CC BY). http://creativecommons.org/licenses/by/4.0/

(c) (i) Open Access

\section{Abstract}

Nowadays electricity market industry is become a major impact on power system for privatization and deregulation of power in global wise. As per the limitation of the transmission system, the complexity arises and the supply fact will be with demand at the time of balancing. The congestion of the power system is occurring based on the transmission limits of the power desire and amount for operating the system. In order to avoid transmission line congestion, enhanced STF-LODF method is proposed. It shows the regulated line transmission flow with generating units by implementing renewable energy resources (RER) based on enhanced STF-LODF in power systems. It avoids the congestion of transmission line frequently in the power system and manages price based on pricing and sensitivity approach, and also manages optimal location of congestion transmission and instability issues of voltage. The congestion management of Locational Marginal Pricing (LMP) is performed with minimum line loss, less cost, line flow, better sensitivity and better performances in optimal power flow and control flow. The efficiency of the proposed power system is analyzed and verified by the simulation results of tested IEEE 14 bus system.

\section{Keywords}

LODF Factor, Sensitivity, LMP, Congestion Management, Optimal Power Flow, Renewable Resource

\section{Introduction}

Throughout the world, Electricity Supply Industry (ESI) is used to utilize the resource service at competitive

${ }^{*}$ Corresponding author. 
prices for customer by restructuring. It eliminates the generation monopoly and sector trade at any possible level. By restricting the advantages will increase the market share to win and continue with the factor of competitive. The objectives make them efficient process to supply the stochastic and the function of demand in the environment. It provides better results of returns to the customer even when it is in risk of congestion occurrences. It occurs in the line of transmission or overloaded and from generator the system operators are prevented from the power of additional dispatching.

Locational Marginal Pricing (LMP) is an approach for the system transmission to have efficient process and management during the occurrences of congestion. It arises for the economic privations with restrictions. In certain location, the demand of electricity will occur due to the limited capacity of power carrying. The constraint of transmission includes the process for the power supply and based on congestion, the supply of power at various locations is performed. As per the supply the charge is increased to the customer.

The vital mechanism of transmission network is based on the electricity supply to the customer in the economical power markets. Day to day it increases based on population and enhances the system to establish the load demand to meet the needs with costliest choice. It prevents the limits of supply, monopolies and provides choices to the customer to choose the utility package. By coordinates lack the occurrences of congestion are made between the utilities of transmission and generation. Dispatch of all transaction is not being possible by the congestion occurrences. For the management process of the congestion, the FACTS device TCSC is placed in the series. The optimal location finding is sensitive approach to place the device. The loss of total reactive power can reduce by the placement of devices in the optimal location. Figure 1 shows the power system simplified interconnection between buses.

The static management of congestion is optimizing the location to place the device with the index of overloaded sensitivity factor. Transmission Line Relief (TLR) method is used to manage the congestion in power deregulating. As per the congestion and flow based pricing, the transmission pricing is evaluated. By congestion allocation charges, the scheduling of relieving congestion is done by proper pricing scheme. The loss of transmission is avoided by the congested line appearances. The transfer capacity limits the power transfer based on the factors in the operation of power system. Based on limits of stability, the security criterion is process in the system and it varies from time to time. By low price generating units, the system costs are estimated.

The management of the system categorized to optimize the power system based on generation and load re-dispatch, price-based solutions, optimal system reconfiguration and finally transmission and generation expansion. According to Independent System Operator (ISO), the congestion management is performed with the action as per the priority and the applicability of the classification.

\section{Related Work}

In this section, the literature review related to transmission line congestion and LMP criteria is discussed. For double-sided bidding of optimal strategy is based on the bi-level optimization problem. The issues of lower and upper level of system operator (SO) represent the process of market clearing and the non-linear functions of maximization profit of supplier's. Bacteria foraging optimization (BFO) is obtain the global solution for the issues $[1]$.

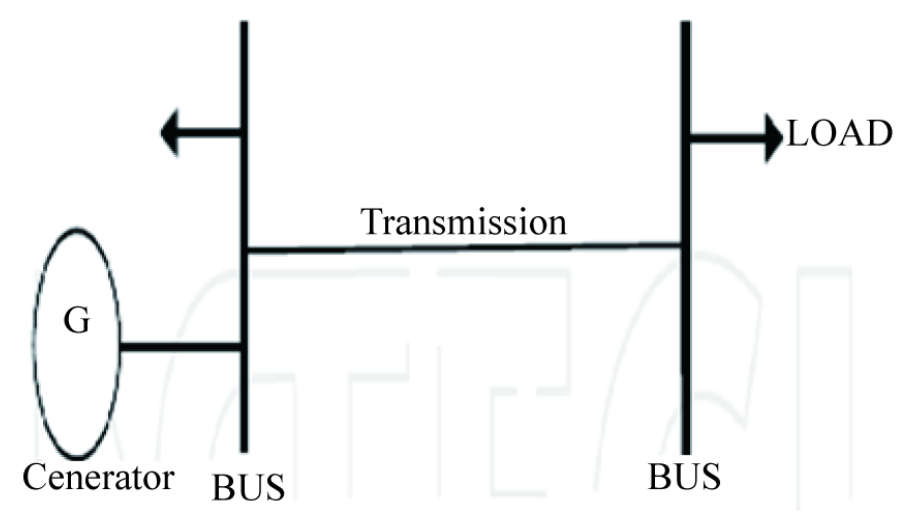

Figure 1. Interconnected power system. 
In cellular network the multi hop cognitive cellular network $(\mathrm{MC} 2 \mathrm{~N})$ architecture is facilitate the transmission of data exploding with minimum energy consumption. The maximum independent set (MIS) is formulated the optimization problem with energy consumption [2]. The mobile network will be in contacts between nodes and ensure the reliability of transmitting data between the source and destination. However, the opportunistic networks ineffectiveness is based on the modified versions of TCP [3]. To generate global solution the integer linear program (ILP) is used in the static and dynamic method to make decision based on throughput instantaneous. The congestion window management is used to manage the optimal performances of reconfiguration. Based on the renewal theory the modeling of the CMT session expected throughput is processed and Markov chain used for others. The Markov chain provide more accurate and endure with the issues of scalability. But renewal model provide more cost effective with less accurate [4].

The Locational Marginal Pricing (LMP) approach is used to monitor and find the transmission congestion in the power system. It is used to relieve the congestion and minimize the variations by the criteria of N-1 security. In wind farms application, the nature of the speed of wind is determined by the location finding and appropriate size. The probability of results is utilized the sizing and the placement of wind [5]. The LMP approach is used to utilize the system for congestion location adopting under the system significant conditions like efficiency of congestion minimizing, failure generation, increasing load and outage of transmission line [6].

To determine the line influential the transmission switching (TS) is originated the issues of optimization for disconnection. It is embedded with the binary variable and AC constraints for congestion relieve without the security of violating voltage in the issues of optimal power flow (OPF). The issue of mixed-integer nonlinear programming (MINLP) is solved by Benders decomposition. It also formulated based on DC optimal power flow (DCOPF) and by simplified models shortcomings the collapse of voltage is occur in some cases [7]. The RSCUC is based on load ability set notion and generate large set and demand. The residual demand scenarios met the capability of transmission and reserve of power system at specific hour [8].

A transmission operator (TO) is used to make a decision in network transmission investment, at the same time as expect the electricity market outcome with the level of operating and determine the sales and amount. By bi-level programming models the issues of transmission investment in upper level model is represented by TO. It is reduced to mixed-integer linear and nonlinear programming based on the optimality conditions of KarushKuhn-Tucker (KKT) and duality theory [9].

In order to manage the transmission congestion and the issues of instability voltage the FACTS devices is placed in the optimal location by using the pricing and sensitivity approach. This method is simulated the approach and verified the results by testing it in modified IEEE 14 bus system [10]. As well as, the best location is identified using the list of priority to have a minimum rent of total congestion and creation cost for TCSC. The simulated result is verified by testing using power world simulator 11.0 in modified IEEE 9 bus system [11].

For minimum cost and the maximum LM the pool model is formulated based on the novel multi-objective with its objective functions. The best tradeoff is achieved among the LM and cost based on Non-dominated Sorting Genetic Algorithm II (NSGAII). It provides minimum cost for the management of congestion and stability maintaining in the line transmission as improve congestion [12].

In restructured power market LMP is used to address the price of congestion based on Genetic Algorithm (GA) based DCOPF model. It includes the loss of distributed and concentrated model in the system in order to avoid the mismatch at the slack bus. LMP decomposition is decomposing into price such as loss, energy and congestion price. It is applied for bus system New England 39 bus, IEEE 14 bus and Indian Power System 75 bus [13]. In AC optimal power flow is developed with the LMP decomposition and ensures the adequacy revenue in the settlement of FTR settlement. It utilizes the optimization problem with constraints by the simple quadratic programming [14].

The LMP-financial transmission right (FTR) mechanisms are the capability of regulating network with the occurrence of high voltage dc (HVDC) lines. The LMP-FTR mechanism framework establishment believes the regulation of the system with fully AC and unregulated of the system is through proxy-transaction bids. However, the flow of the system is management and maintained [15].

\section{Proposed Work}

In this section the proposed approach is explained with its implementation in the power system. The Enhanced STF-LODF is proposed with the efficiency of the system, minimum line loss, better sensitivity, less cost, line 
flow, and better performances in optimal power flow and control flow. In power system Renewable Energy Resources (RER) is frequently used and it is installed based on the distribution factor of line outage. By the wind nature probalistic the system is utilized with the rapid increase of power consumption and the transmission management of congestion is out of control. In order to avoid this and to improve efficiency the Enhanced STF-LODF method is proposed by enhancing the Shift tolerant factor (STF) with the line outage distribution factor (LODF). As shown in Figure 2, the proposed approach flow work is performed in the power system.

Shift Tolerant factor is used to inject the bus power and to determine the tolerant factor of the power system with the changes. LODF is used to changes the line flow by the line outage and determine the changes from the pre-contingency flow to post-contingency flow with the occurrences of congestion. It is a real time process of the power system based on network topology.

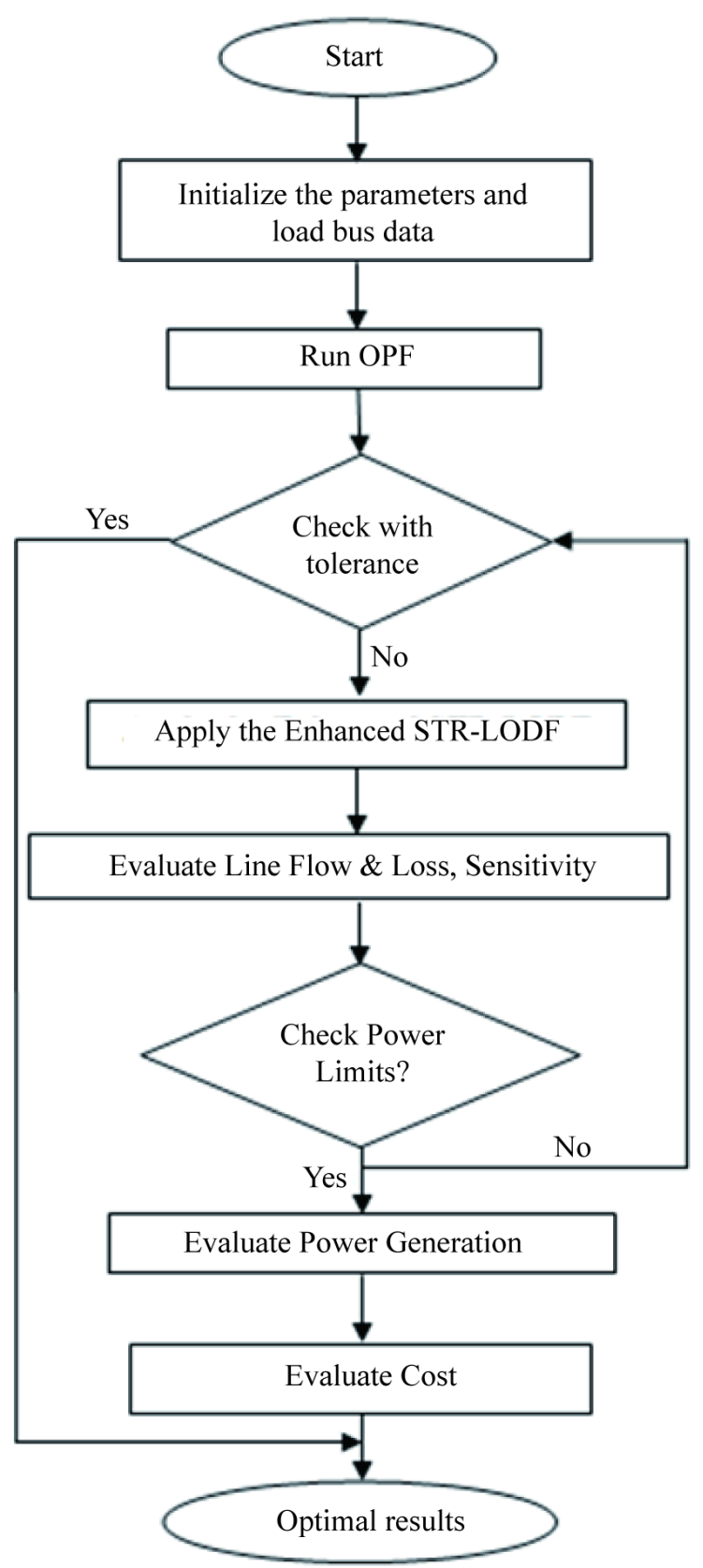

Figure 2. The proposed Enhanced STF-LODF Flow work. 
As per the flow work of the proposed frame work the optimal power flow is formulated. In order to evaluate the schedule of load, pricing, congestion management and the generation dispatch the Optimal Power Flow (OPF) is used in the pool-based deregulated electricity markets. Based on optimal solution of nonlinear constraints of equality and inequality objective functions the network constraints of load and cost is estimated. It stated as:

$$
\operatorname{Min} \sum_{i=1}^{G_{n}} a_{i} \times\left(P_{G i}\right)^{2}+b_{i} \times P_{G i}+c_{i}
$$

where, $G_{n}$ is represent the number of generating units; the coefficient of cost is denoted as $a_{i}, b_{i}$ and $c_{i} \times P_{G i}$ represent the power generator output. The constraints of power balance of the bus with the power losses network and the line congestion is evaluated as given below.

$$
\sum_{i} P_{G i}-P_{L}\left(P_{G i}\right)=\sum_{j} P_{D j}
$$

The generator power output, the real and reactive power demand, the capability of the power transfer and the voltage limits is stated as given below:

$$
\begin{gathered}
P_{G i}^{\min } \leq P_{G i} \leq P_{G i}^{\max } \\
Q_{G i}^{\min } \leq Q_{G i} \leq Q_{G i}^{\max } \\
P_{D j}^{\min } \leq P_{D j} \leq P_{D j}^{\max } \\
V_{k}^{\min } \leq V_{k} \leq V_{k}^{\max } \\
\text { Flow }_{m}^{\min } \leq \text { Flow }_{m} \leq \text { Flow }_{m}^{\max } \\
\text { Con }_{l}=\frac{P_{i j}^{\prime}}{P_{i j}}
\end{gathered}
$$

where, $\operatorname{con}_{l}$ is representing the congestion in per line; $P_{i j}^{\prime}$ denotes the flow of power between the buses and $V_{k}$ denotes the voltage limits of $k$ buses. The objective function $(O F)$ of line congestion is stated below.

$$
O F=\left\|\frac{P_{i j}^{\prime}}{P_{i j}}\right\|_{\alpha}
$$

The function of proposed method factors is evaluated the power flow and the line flow between the lines is stated below. The STF factor $(T F)$ is evaluated to provide effectiveness and impedance factor to define the distribution of power transfer and coefficient. The distributed factors of the outage line is redistributed in the system as $L O D F_{l, k}$.

$$
\begin{gathered}
T F=\left(\frac{r_{A}+r_{O}}{\sqrt{2}\left(r_{B}+r_{O}\right)}\right) \\
L O D F_{l, k}=\frac{N_{k} \cdot x_{k}}{N_{1} \cdot x_{1}} \cdot \frac{\left(x_{l r}-x_{l s}-x_{m r}+x_{m s}\right)}{N_{k} \cdot x_{k}-\left(x_{r r}-x_{s s}-2 x_{r s}\right)} \\
\Delta P_{1} \approx L O D F_{l, k} \times P_{k} \times T F
\end{gathered}
$$

where, $\Delta P_{1}$ denotes the flow of line change due to the outage of like $k ; P_{k}$ represent the contingency of line flow. If $P_{k}=100 \mathrm{mw}$ then the $P_{1}=50 \mathrm{mw}$ due to the outage of line.

If $L O D F_{l, k}=0.1$ then the flow of contingency line $\Delta P_{1}=10 \mathrm{mw}\left(L O D F_{l, k} \times P_{k}=0.1 \times 100\right)$. The sensitivity of the line transmission congestion is evaluated by the function given below. Also, the transmission line excess of flow of power is stated below.

$$
S_{i j}^{k}=\frac{\overline{\Delta P_{i j}}}{\Delta P_{k}}
$$




$$
\overline{\Delta P_{i j}}=P_{i j}-\overline{P_{i j}}
$$

where, $P_{i j}$ denotes the actual power in the transmission line and the power flow limit is represented as $\overline{P_{i j}}$. The procedure of the proposed approach is performed in the power system as given below.

Procedure of Enhanced STF-LODF method:

1. Initialization of bus data with its parameters like number of buses and lines, coefficients, min and max limits of power generator.

2. Randomly generating of power generator with decodes function to evaluate the power in the system by using function (2).

3. Apply the proposed algorithm to generate power with the LMP concept using the function (12) and find the optimal location of the system to place LMP.

4. Apply the factor of shift tolerant to generate shift factors and to define the coefficient of the bus power system by using function (10).

5. Apply the line outage distribution factor to find changes of line and the outage of the power system by using the function (11).

6. Placing of line congestion (LMP) in the optimal location.

7. Evaluate the line flow, line loss, power flow and the power loss. Also find the sensitivity of the system using the function $(13-14)$

8. Continue step 2 to generate the power function with and without loss case. With the limits of power.

9. Evaluate the cost of the power system using the function (1).

10. Evaluate the optimal results of the proposed system.

\section{Performance Analysis}

In this section, the simulation of the proposed Enhanced STF-LODF is implemented and evaluated the results by simulating the approach in MATLAB/SIMULINK platform in IEEE bus system. The performances analysis of the proposed approach results shows the better performances than the existing approach with less cost, low power loss, better sensitivity and improvement in avoiding congestion and management of the system. Figure 3 shows the Standard IEEE 14 bus system structure.

Table 1 shows the input data of fuel cost coefficient. Table 2 illustrates the results of proposed Enhanced STF-LODF approach line flow and line loss. Table 3 shows the generating of power for post and pre installation of proposed approach in the bus system. It illustrate the real and reactive power generation of the proposed system and the pre installation of the approach. The analysis of the obtained results shows the improvement in generating power.

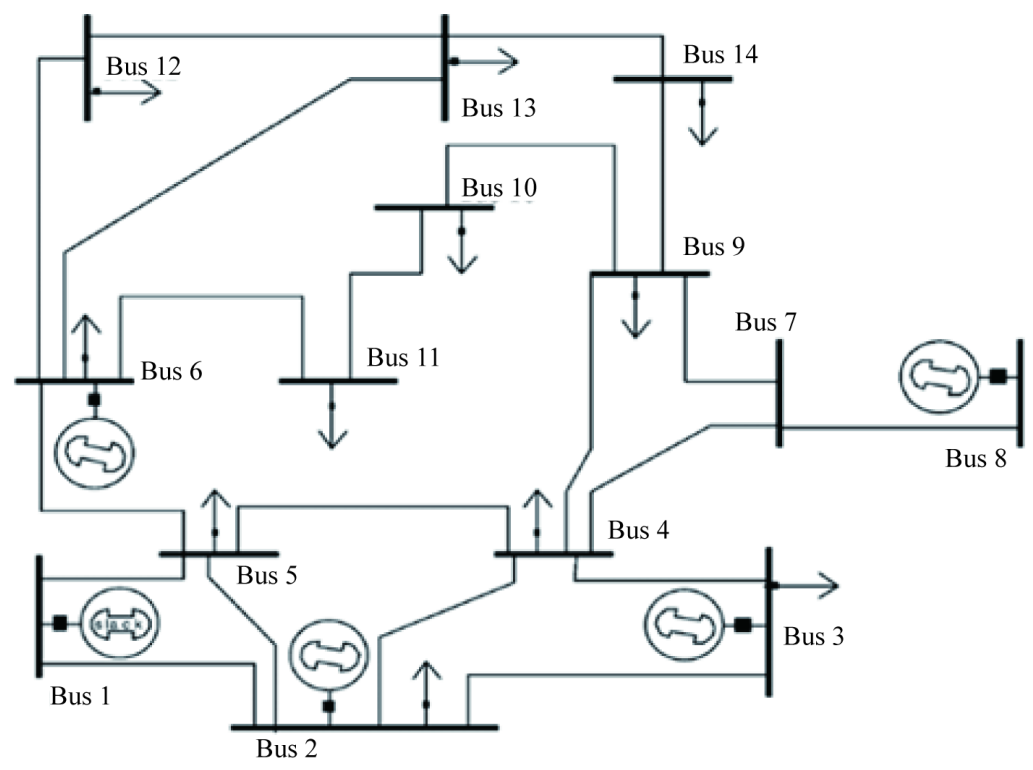

Figure 3. Standard IEEE 14 bus system. 
Table 1. Fuel cost coefficients-input data.

\begin{tabular}{cccc}
\hline Bus No. & $\mathrm{a}(\$ / \mathrm{MW} 2 \mathrm{~h})$ & $\mathrm{b}(\$ / \mathrm{MWh})$ & $\mathrm{C} / \mathrm{h})$ \\
\hline 1 & 0.0252 & 16 & 0 \\
2 & 0.1400 & 14 & 0 \\
3 & 0.5000 & 8 & 0 \\
6 & 0.0667 & 26 & 0 \\
8 & 0.2000 & 24 & 0 \\
\hline
\end{tabular}

Table 2. Analysis of line flow and line loss of proposed enhanced STF-LODF approach.

\begin{tabular}{|c|c|c|c|c|c|}
\hline \multirow{2}{*}{ From bus } & \multirow{2}{*}{ To bus } & \multicolumn{2}{|c|}{ Line Flow } & \multicolumn{2}{|c|}{ Line Loss } \\
\hline & & MW & MVar & MW & MVar \\
\hline 1 & 2 & 157.080 & 157.080 & 4.309 & 13.155 \\
\hline 1 & 5 & 75.513 & 75.513 & 2.773 & 11.445 \\
\hline 2 & 3 & 73.396 & 73.396 & 2.333 & 9.830 \\
\hline 2 & 4 & 55.943 & 55.943 & 1.670 & 5.067 \\
\hline 2 & 5 & 41.733 & 41.733 & 0.920 & 2.809 \\
\hline 3 & 4 & -23.137 & -23.137 & 0.391 & 0.998 \\
\hline 4 & 5 & -59.585 & -59.585 & 0.479 & 1.511 \\
\hline 4 & 7 & 27.066 & 27.066 & 0.000 & 1.932 \\
\hline 4 & 9 & 15.464 & 15.464 & 0.000 & 1.292 \\
\hline 5 & 6 & 45.889 & 45.889 & 0.000 & 5.774 \\
\hline 6 & 11 & 8.287 & 8.287 & 0.123 & 0.257 \\
\hline 6 & 12 & 8.064 & 8.064 & 0.081 & 0.168 \\
\hline 6 & 13 & 18.337 & 18.337 & 0.252 & 0.496 \\
\hline 7 & 8 & -0.000 & -0.000 & 0.000 & 0.668 \\
\hline 7 & 9 & 27.066 & 27.066 & -0.000 & 0.957 \\
\hline 9 & 10 & 4.393 & 4.393 & 0.006 & 0.016 \\
\hline 9 & 14 & 8.637 & 8.637 & 0.089 & 0.190 \\
\hline 10 & 11 & -4.613 & -4.613 & 0.051 & 0.120 \\
\hline 12 & 13 & 1.884 & 1.884 & 0.011 & 0.010 \\
\hline 13 & 14 & 6.458 & 6.458 & 0.105 & 0.215 \\
\hline
\end{tabular}

Table 3. Pre and post installation of enhanced STF-LODF power generation.

\begin{tabular}{|c|c|c|c|c|c|c|c|}
\hline \multirow{2}{*}{ Bus } & \multicolumn{2}{|c|}{ Load } & \multirow{2}{*}{$\begin{array}{l}\text { Rated Voltage } \\
\text { (pu) }\end{array}$} & \multicolumn{2}{|c|}{ Generation(Pre) } & \multicolumn{2}{|c|}{ Generation(Post) } \\
\hline & MW & MVar & & MW & MVar & MW & MVar \\
\hline 1 & 0.000 & 0.000 & 1.0600 & 0.000 & 0.000 & 0.000 & 0.000 \\
\hline 2 & 21.700 & 12.700 & 1.0450 & 21.700 & 12.700 & 21.700 & 12.700 \\
\hline 3 & 94.200 & 19.000 & 1.0100 & 94.200 & 19.000 & 94.200 & 19.000 \\
\hline 4 & 47.800 & -3.900 & 1.0132 & 47.800 & -3.900 & 47.800 & -3.900 \\
\hline 5 & 7.600 & 1.600 & 1.0166 & 7.600 & 1.600 & 7.600 & 1.600 \\
\hline 6 & 11.200 & 7.500 & 1.0700 & 11.200 & 7.500 & 11.200 & 7.500 \\
\hline 7 & 0.000 & 0.000 & 1.0457 & 0.000 & 0.000 & 0.000 & 0.000 \\
\hline 8 & 0.000 & 0.000 & 1.0800 & 0.000 & 0.000 & 0.000 & 0.000 \\
\hline 9 & 29.500 & 16.600 & 1.0305 & -106.250 & -289.343 & -113.090 & -304.758 \\
\hline 10 & 9.000 & 5.800 & 1.0299 & 9.000 & 5.800 & 9.000 & 5.800 \\
\hline 11 & 3.500 & 1.800 & 1.0461 & 3.500 & 1.800 & 3.500 & 1.800 \\
\hline 12 & 6.100 & 1.600 & 1.0533 & 6.100 & 1.600 & 6.100 & 1.600 \\
\hline 13 & 13.500 & 5.800 & 1.0466 & -97.198 & -227.146 & -104.594 & -242.709 \\
\hline 14 & 14.900 & 5.000 & 1.0193 & 271.000 & 539.401 & 280.966 & 560.198 \\
\hline
\end{tabular}


In Table 4, the factor matrix of 14 bus system sensitivity is illustrated and an obtained result is analyzed. Figure 4 shows the performance analysis of bus Vs voltage results. Table 5 shows the analysis of various algorithm of power system.

Table 6 shows the performances analysis of proposed power system with load, loss and cost of the system. As well as Figure 5 shows the analysis of bus Vs real power of the proposed system and Figure 6 shows the analysis of bus vs. reactive power of the system.

Table 4. Analysis of sensitivity factor matrix for 14 bus system.

\begin{tabular}{|c|c|c|c|c|c|c|c|c|c|c|c|c|c|c|}
\hline Bus & 1 & 2 & 3 & 4 & 5 & 6 & 7 & 8 & 9 & 10 & 11 & 12 & 13 & 14 \\
\hline 1 & 0 & 0 & 0 & 0 & 0 & 0 & 0 & 0 & 0 & 0 & 0 & 0 & 0 & 0 \\
\hline 2 & 0 & 0.0496 & 0.0442 & 0.0395 & 0.0361 & 0.0373 & 0.0389 & 0.0389 & 0.0386 & 0.0383 & 0.0378 & 0.0374 & 0.0375 & 0.0381 \\
\hline 3 & 0 & 0.0442 & 0.1495 & 0.0695 & 0.0565 & 0.0609 & 0.0671 & 0.0671 & 0.0659 & 0.0650 & 0.0630 & 0.0613 & 0.0616 & 0.0640 \\
\hline 4 & 0 & 0.0395 & 0.0695 & 0.0954 & 0.0742 & 0.0814 & 0.0916 & 0.0916 & 0.0896 & 0.0881 & 0.0848 & 0.0820 & 0.0825 & 0.0865 \\
\hline 5 & 0 & 0.0361 & 0.0565 & 0.0742 & 0.0869 & 0.0825 & 0.0764 & 0.0764 & 0.0776 & 0.0785 & 0.0805 & 0.0822 & 0.0819 & 0.0795 \\
\hline 6 & 0 & 0.0373 & 0.0609 & 0.0814 & 0.0825 & 0.2485 & 0.1268 & 0.1268 & 0.1506 & 0.1680 & 0.2075 & 0.2407 & 0.2347 & 0.1874 \\
\hline 7 & 0 & 0.0389 & 0.0671 & 0.0916 & 0.0764 & 0.1268 & 0.2242 & 0.2242 & 0.1839 & 0.1738 & 0.1507 & 0.1313 & 0.1348 & 0.1625 \\
\hline 8 & 0 & 0.0389 & 0.0671 & 0.0916 & 0.0764 & 0.1268 & 0.2242 & 0.4003 & 0.1839 & 0.1738 & 0.1507 & 0.1313 & 0.1348 & 0.1625 \\
\hline 9 & 0 & 0.0386 & 0.0659 & 0.0896 & 0.0776 & 0.1506 & 0.1839 & 0.1839 & 0.2336 & 0.2188 & 0.1853 & 0.1572 & 0.1623 & 0.2024 \\
\hline 10 & 0 & 0.0383 & 0.0650 & 0.0881 & 0.0785 & 0.1680 & 0.1738 & 0.1738 & 0.2188 & 0.2793 & 0.2246 & 0.1720 & 0.1752 & 0.1998 \\
\hline 11 & 0 & 0.0378 & 0.0630 & 0.0848 & 0.0805 & 0.2075 & 0.1507 & 0.1507 & 0.1853 & 0.2246 & 0.3140 & 0.2058 & 0.2044 & 0.1937 \\
\hline 12 & 0 & 0.0374 & 0.0613 & 0.0820 & 0.0822 & 0.2407 & 0.1313 & 0.1313 & 0.1572 & 0.1720 & 0.2058 & 0.3738 & 0.2778 & 0.2099 \\
\hline 13 & 0 & 0.0375 & 0.0616 & 0.0825 & 0.0819 & 0.2347 & 0.1348 & 0.1348 & 0.1623 & 0.1752 & 0.2044 & 0.2778 & 0.3116 & 0.2276 \\
\hline 14 & 0 & 0.0381 & 0.0640 & 0.0865 & 0.0795 & 0.1874 & 0.1625 & 0.1625 & 0.2024 & 0.1998 & 0.1937 & 0.2099 & 0.2276 & 0.3656 \\
\hline
\end{tabular}

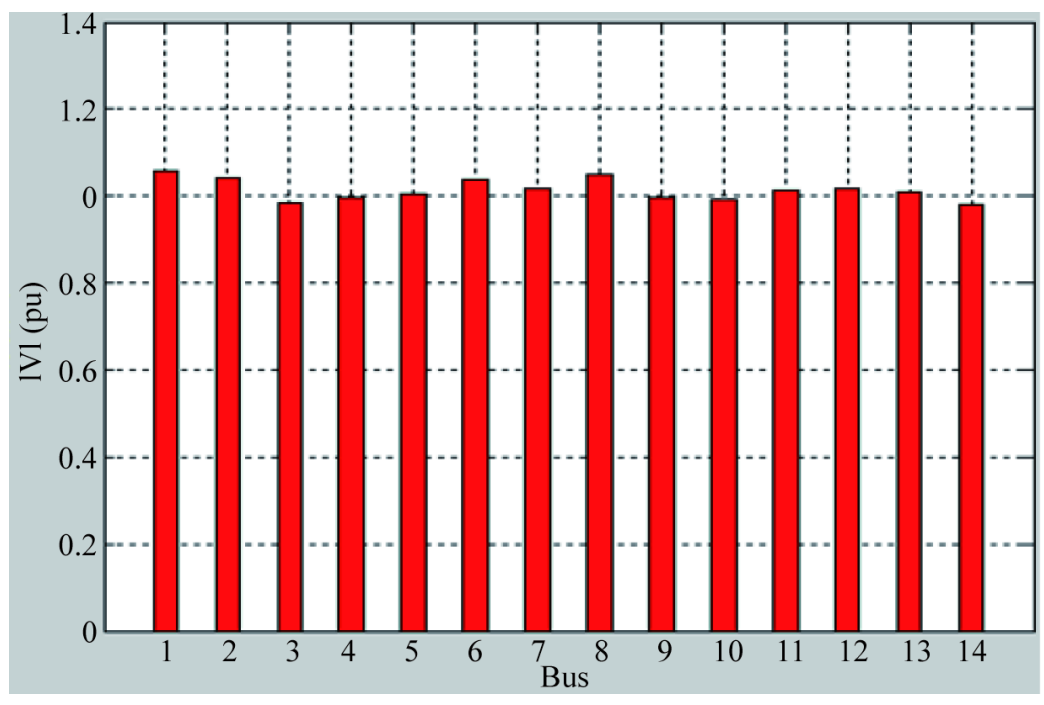

Figure 4. Analysis of bus vs. voltage.

Table 5. Performances analysis of various algorithm of the power system.

\begin{tabular}{cc} 
Algorithm & Power Loss \\
\hline TCSC (Thyristor Controlled Series Capacitors) & 158.052 \\
LP based DCOPF approach & 130.247 \\
GA (Genetic Algorithm) approach & 63.725 \\
RED (Relative Electrical Distances) & 19.79 \\
Proposed (Enhanced STF-LODF) & 13.593 \\
\hline
\end{tabular}


Table 6. Performances analysis of proposed system.

\begin{tabular}{ccc}
\hline S. No & Description & Values \\
\hline 1 & Total Generation (Post) & $264.382 \mathrm{MW}, 58.831 \mathrm{MVar}$ \\
2 & Total Generation (Pre) & $268.651 \mathrm{MW}, 69.012 \mathrm{MVar}$ \\
3 & Total Load & $259.000 \mathrm{MW}, 73.500 \mathrm{MVar}$ \\
4 & Total Losses & $13.593 \mathrm{MV}, 56.910 \mathrm{MVar}$ \\
5 & Total Cost & 5859.7 \\
\hline
\end{tabular}

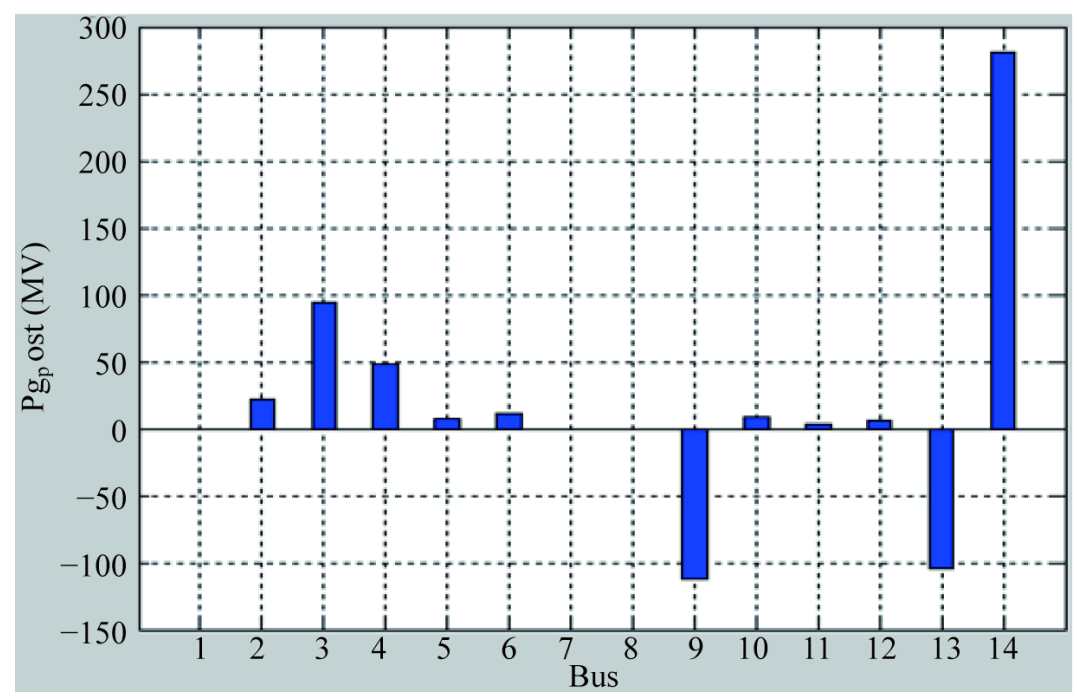

Figure 5. Analysis of bus vs. real power.

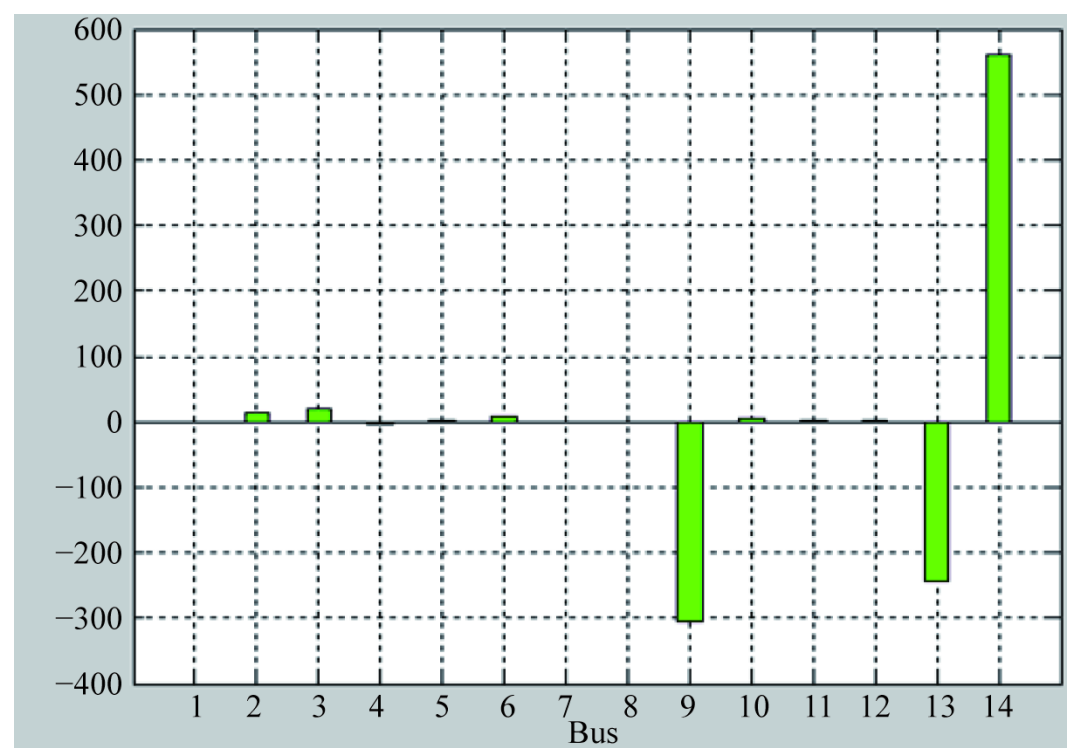

Figure 6. Analysis of bus vs. reactive power.

\section{Conclusions}

In this paper, an enhanced STF-LODF is proposed for solving the issues of congestion in OPF. The initial solution of the proposed system is provided with improvement in congestion management and avoiding it in line transmission. The proposed system model is tested in standard IEEE 14 bus system. By using proposed algo- 
rithm, the power system provides less power and cost with efficient optimization process to obtain optimal solution. The sensitivity of the system is improved with the best solution in the proposed power system. By the proposed algorithm, the cost estimation shows the reduction with congestion process of the system than the existing algorithm. The performances analysis and comparison of proposed simulation results show better performances than the existing system.

In future work, power system improvement is essential and enhances the function of congestion with the parameters like accuracy, the resolving solution for the issues and robustness. Propose or improve the approach minimize cost and loss and enhance speed process with efficient control process in network.

\section{References}

[1] Jain, A.K., Srivastava, S.C., Singh, S.N. and Srivastava, L. (2015) Bacteria Foraging Optimization Based Bidding Strategy under Transmission Congestion. IEEE Systems Journal, 9, 141-151.

[2] Li, M., Li, P., Huang, X.X., Fang, Y.G. and Glisic, S. (2015) Energy Consumption Optimization for Multi Hop Cognitive Cellular Networks. IEEE Transactions on Mobile Computing, 14, 358-372.

[3] Soelistijanto, B. and Howarth, M.P. (2014) Transfer Reliability and Congestion Control Strategies in Opportunistic Networks: A Survey. IEEE Communications Surveys \& Tutorials, 16, 538-555.

[4] Wallace, T.D. and Shami, A. (2014) Concurrent Multipath Transfer Using SCTP: Modelling and Congestion Window Management. IEEE Transactions on Mobile Computing, 13, 2510-2523.

[5] Ahmadi, H. and Lesani, H. (2014) Transmission Congestion Management through LMP Difference Minimization: A Renewable Energy Placement Case Study. Arabian Journal for Science and Engineering, 39, 1963-1969.

[6] Ramachandran, P. and Senthil, R. (2010) Locational Marginal Pricing Approach to Minimize Congestion in Restructured Power Market. Journal of Electrical and Electronics Engineering Research, 2, 143-153.

[7] Khanabadi, M., Ghasemi, H. and Doostizadeh, M. (2013) Optimal Transmission Switching Considering Voltage Security and N-1 Contingency Analysis. IEEE Transactions on Power Systems, 28, 542-550.

[8] Kalantari, A., Restrepo, J.F. and Galiana, F.D. (2013) Security-Constrained Unit Commitment with Uncertain Wind Generation: The Loadability Set Approach. IEEE Transactions on Power Systems, 28, 1787-1796.

[9] Jenabi, M., Mohammad, S., Ghomi, T.F. and Smeers, Y. (2013) Bi-Level Game Approaches for Coordination of Generation and Transmission Expansion Planning within a Market Environment. IEEE Transactions on Power Systems, 28, 2639-2650.

[10] Rao, J.S. and Amarnath, Dr.J. (2013) Transmission Congestion Management Comparative Studies in Restructured Power System. International Journal of Scientific \& Engineering Research, 4, 8.

[11] Sambasivarao, N., Amarnath, J. and Purnachandrarao, V. (2013) Congestion Management Using Facts Devices in Deregulated Power System. IJRET: International Journal of Research in Engineering and Technology, 2, 90-95.

[12] Bhattacharjee, T. and Chakraborty, A.K. (2012) Congestion Management in a Deregulated Power System Using NSGAII. 2101 IEEE 5th, 1-6.

[13] Matcha, M., Matam, S.K. and Maheswarapu, S. (2012) LMP Calculation with Distributed Loss Using GA Based DCOPF. Journal of Electrical Systems, 8, 292-303.

[14] Sarkar, V. and Khaparde, S.A. (2011) Optimal LMP Decomposition for the ACOPF Calculation. IEEE Transactions on Power Systems, 26, 1714-1723.

[15] Sarkar, V. and Khaparde, S.A. (2008) Implementation of LMP-FTR Mechanism in an AC-DC System. IEEE Transactions on Power Systems, 23, 737-746. 


\section{Submit or recommend next manuscript to SCIRP and we will provide best service for you:}

Accepting pre-submission inquiries through Email, Facebook, LinkedIn, Twitter, etc.

A wide selection of journals (inclusive of 9 subjects, more than 200 journals)

Providing 24-hour high-quality service

User-friendly online submission system

Fair and swift peer-review system

Efficient typesetting and proofreading procedure

Display of the result of downloads and visits, as well as the number of cited articles

Maximum dissemination of your research work

Submit your manuscript at: http://papersubmission.scirp.org/ 